\title{
Psychoemotional States and Personality Traits of Patients Undergoing Treatment
}

\author{
Maksimenkova Larisa I. ${ }^{1, *}$ Guchkova Tatyana N. ${ }^{1}$ Chernova Veronika ${ }^{1}$ \\ Nikolaev Sergey M. ${ }^{1}$ Romanov Kirill I. ${ }^{2}$ Rantsev Gennady M. ${ }^{1}$
}

\author{
${ }^{1}$ Pskov State University, 180000, 2 Lenin square, Pskov, Russia \\ ${ }^{2}$ Academy of Law Management of the Federal Penal Service of Russia, Campus in Pskov, 180000, 28 Zonal Highway, \\ Pskov, Russia \\ *Corresponding author.Email: lora_max@mail.ru
}

\begin{abstract}
A rapidly accelerating pace of life, the need for more frequent and close contacts between people, and abundance of information received lead to the inevitable occurrence of stressful situations which require the neuropsychic stability of the individual. Premorbid personality traits can act either as stabilization factors and contribute to the adaptation of the individual to reality, or support personality disorganization and lead to disintegration and maladjustment. Thus, the depletion of the personality's adaptive resources causes the emergence of pathological psychoemotional states. At present, the most frequent negative emotional states that a person encounters are the experience of loneliness, depressive and neurotic states. Of particular interest, in our opinion, is the analysis of severity of the emotional states in respondents of the clinical and non-clinical (conventionally healthy) samples. This paper presents the analysis of pathological emotional states of the individual (experience of loneliness, depressive and neurotic states) as a cause of disorders in adaptation and socialization of the individual, as well as the analysis of personality traits in patients undergoing treatment compared to those who have never sought medical help for mental health problems. The analysis of data obtained in the empirical study revealed significant differences between hospital patients $(n=72)$ and conventionally healthy individuals $(n=95)$ in terms of their personality traits in the form of sharpening of hypochondriacal, paranoia and schizoid manifestations, a tendency to hysteroid reactions, etc. In addition, significantly higher indicators of psychoemotional state were observed, namely: depressive experience of varying degrees of severity, high levels of various aspects of the experience of loneliness and indicators of neurotization and psychopathization.
\end{abstract}

Keywords: psychoemotional state, loneliness, depression, psychopathization, neurotization

\section{INTRODUCTION}

A rapidly accelerating pace of life, frequent and close contacts between people, and abundance of information received have a significant impact on the psychoemotional state of the individual, cause neuropsychic stress, and deplete the adaptive resources of the individual.

Thus, the current situation in society can contribute to the manifestation of psychoemotional states that provoke the emergence of both functional and neuropsychic disorders.

Psychoemotional states are a special form of human mental state and experience with the manifestation of emotional responses to oneself, others, and to reality. These states are largely regulated by the emotionalvolitional sphere and include emotional responses and relationships. The concept of 'psychoemotional state' is collective and includes a number of phenomena, such as loneliness (Kon, Korchagina, Rogova, Fromm-Reichman,
Frankl), depression (Aleksandrovsky, Vertogradova, Lakosina, Sinitsyn \& Smulevich), and neurosis (Vasilyuk, Garbuzov, Karvasarsky, Myasishchev, Svyadosch, Horney).

Premorbid personality traits can act either as stabilization factors and contribute to the adaptation of the individual, or support personal disorganization and lead to disintegration and maladjustment.

A psychologist needs information about the factors contributing to the onset of psychoemotional states in order to more effectively organize preventive and corrective activities.

The study suggests the hypothesis that there are differences between patients undergoing hospital treatment and those who have never sought medical help for mental health problems in terms of their psychoemotional state and personality traits. 


\section{PROBLEM STATEMENT}

Psychoemotional state is the dominance of one or another emotional reaction arising in response to external impact. This is a variety of phenomena formed by self-reflection, integration into the environment, which are accompanied by an exacerbation of reflection and changes in the emotional sphere.

The formation of neurotic reactions in the individual (depression, anxiety, fear, somatoform disorders), which become hypertrophied in unfavorable circumstances and can provoke disturbances in the system of significant relationships. As a result, the views and assessments of the subject change, his own personality is perceived differently, and ideas about his place in society change [4]. This causes disorders in adaptation and socialization of the individual [8].

According to the pathogenetic theory of neuroses proposed by V.N. Myasishchev, personality is a system of relationships, which can be violated depending on the conditions of development. This violation is the essence of neurosis which affects significant relationships with a pronounced emotional and motivational-behavioral component [10]. Neurosogenesis is a change in the functional state of the individual as a result of violations in the system of significant relationships (Airapetyants, Vasilyuk, Zakharzhevsky, Zlokazova \& Martovetskaya, Mendelevich, Myasishchev, Svyadosch, Ushkalova), which causes physical and psychological maladjustment, that is, a frustrating personality.

The most frequent negative emotional states that a person encounters are depressive experiences and experience of loneliness. The analysis of the problem of loneliness in domestic and foreign literature is considered in various aspects [11].

All over the world, people experience loneliness more often and more acutely, everyone perceives and evaluates loneliness in his own way, based on subjective life experience. For the personality, the feeling of loneliness seems to be a purely individual rather than a general problem [6].

Despite the interdisciplinary nature of the study of the problem of loneliness (philosophical approach - Berdyaev, Starovoitova, Fromm, Yurchenko; sociological Aleinikova, Klimentyev, Puzanova; psychological Bakaldin, Hasanova \& Omarova, Dimitryuk, Kon, Rogova, Slobodchikov, Kharash), the phenomenon of loneliness does not have a single interpretation, and the psychological definition of this phenomenon needs a clear definition [12].

In psychological dictionaries, loneliness is interpreted as one of the psychogenic factors that affects the emotional state and mental health of a person. Isolation (physical or emotional) is crucial for its occurrence, which leads to an increased need for communication.

According to the WHO, depressive states and experiences are one of the key problems of psychiatry and psychology which are caused by a wide variety of factors [9].
According to ICD-10, the depressive episode is characterized by the decreased mood and energy, loss of interests, pronounced exhaustion, a pessimistic assessment of oneself, and one's future and present. Decreased mood can be masked by irritability, exacerbation of phobic or obsessive-compulsive symptoms, hysterical or hypochondriacal manifestations.

Modern psychiatry associates the etiology of this mental disorder with the effect of a combination of biological, psychological and social factors on the individual.

Being one of the main problems of modern society, depressive disorders require careful analysis, development and testing of diagnostic, therapeutic and preventive measures (Aleksandrovsky, Veltischev, Vertogradova, Garanyan, Krasnov, Lakosina, Pilyagina, Smulevich, Stepanov, Tikhonov).

Of particular interest are the issues of preventing depressive personality disorders, identifying risk groups [5], and correcting depressive and anxiety disorders, including self-help methods [1, 2, 7].

The emergence of the considered psychoemotional states (neurosis, loneliness, depression) is mostly due to a complex of triggering mechanisms, including somatic diseases, mental trauma, personality traits, psychological difficulties, the specifics of interpersonal interaction, including virtual one, disorders in the system of significant personality relationships [3]. The manifestations of psychoemotional states are of both psychological and physiological nature; therefore, they damage the physical and mental well-being of the individual.

In this regard, it is of relevence to study and compare the features of these psychoemotional states in patients undergoing treatment and in those who have never sought medical help for mental health problems.

\section{RESEARCH QUESTIONS}

The object of the study is psychoemotional states and personality traits of patients undergoing treatment.

Peculiarities of psychoemotional states (depressive states, experience of loneliness, the level of psychopathization and neurotization) of patients undergoing treatment compared to those who have never sought medical help for mental health problems.

Personality traits of patients undergoing treatment compared to those who have never sought medical help for mental health problems.

\section{PURPOSE OF THE STUDY}

The study aimed to identify and compare psychoemotional states (depressive states, experience of loneliness, the level of psychopathization and neurotization) and personality traits of patients undergoing treatment and those who have never sought medical help for mental health problems. 


\section{RESEARCH METHODS}

The study employed the following methods and techniques:

- Differential questionnaire of experiencing loneliness by E.N. Osin, D.A. Leontyev (Osin, Leontiev, 2013);

- Method for differential diagnosis of depressive states V. Zung (The Zung self-rating depression scale, 1965, adaptation by T. I. Balashova) (Barkanova, 2009);

- Method for the determination of level of neurotization and psychopathization (LNP) by N.F. Burlachuk (Raigorodsky, 2011);

- Mini-Mult (a shortened version of multivariate questionnaire for personality research) (Zaitsev, 2004)

- $\quad$ The resulting data array was subjected to mathematical and statistical processing using descriptive statistics and comparative analysis. Statistical data processing was carried out using the SPSS 21.0 program. The nonparametric Mann-Whitney U-test was used to confirm the statistically significant differences in the MMPI scale indicators of respondents from the clinical and non-clinical samples.

\section{FINDINGS}

The study involved patients undergoing treatment $(n=72)$ and conditionally healthy individuals who had never sought medical help for mental health problems $(n=95)$. The total number of the sample was 167 people.

The Zung self-rating depression scale was employed to study the severity of depressive states in the respondents from clinical and non-clinical samples.

A significant part of respondents from the clinical sample $(75 \%)$ was characterized by depressive experiences of varying severity. In $11.1 \%$ of respondents, pronounced depression was recorded, which indicates depression, sadness, mental and motor inhibition. This state is accompanied by the experience of one's own insignificance, gloomy assessment of the current situation and prospects, the ease of formation of intrapunitive reactions, which can provoke suicidal actions.

On the contrary, the mood of the overwhelming majority $(65.3 \%)$ of respondents from the non-clinical sample was stable, self-esteem, the level of activity, work capacity were high, which indicates the absence of psychological experiences and physiological symptoms of depression. The symptoms of severe depression were not recorded in respondents from the non-clinical sample.

The Zung self-rating depression scale employed in the study revealed a high level of General loneliness $(19.4 \%$ of respondents) and Dependence on communication $(41.7 \%)$ in respondents from the clinical sample, which indicates the severity of feeling of isolation, lack of contacts and emotional closeness to others, perception of oneself as a lonely person.

A significant number $(37.5 \%)$ of respondents showed a low level of Positive loneliness, which indicates the rejection of loneliness, the absence of positive emotions due to loneliness, and the inability to find a resource in loneliness.

On the contrary, in the non-clinical sample, a significantly smaller number of respondents noted a high level of General loneliness (10.5\%), Dependence on communication $(25.3 \%)$ and a low level of Positive loneliness $(29.4 \%)$.

Thus, differences in terms of depression and loneliness were found between patients undergoing treatment and conventionally healthy individuals.

The LNP method used in the study made it possible to identify similar tendencies. A high level of neurotization was recorded in $55.6 \%$ of respondents from the clinical sample, which indicates emotional excitability that can provoke various negative states, such as: anxiety, alarm, confusion, tension, irritability.

At the same time, only $27.7 \%$ had a low level of neurotization, which indicates emotional stability and positive basic experiences. A total of $16.7 \%$ of respondents had indeterminate diagnosis.

A high level of psychopathization was recorded in $23.6 \%$ of patients undergoing treatment, which indicates carelessness and frivolity, rejection of generally accepted moral norms, ease of going beyond morality, cold attitude towards people, stubbornness in interpersonal relationships. Such people are distinguished by the unpredictability of actions and behavior, they tend to provoke conflict situations. A total of $34.7 \%$ of respondents from the clinical sample had indeterminate diagnosis.

However, opposite tendencies were observed in the non-clinical sample. A larger number of respondents $(52.6 \%)$ were characterized by a low level of neurotization, which indicates independence, ease of establishing contacts, self-esteem and positive basic experiences.

High levels of neurotization were noted only in $24.2 \%$ of the non-clinical sample, which is manifested in emotional excitability, dissatisfaction with desires, and frustration. A total of $23.2 \%$ of respondents had indeterminate diagnosis.

On the Pd scale, a high level was recorded in a smaller number of the non-clinical sample compared to the clinical sample $(18.9 \%)$. At the same time, a significant number of respondents from the non-clinical sample $(48.4 \%)$ had indeterminate diagnosis.

To assess neuro-emotional stability, the integration of personality traits and the level of adaptation to the social environment, we used the Mini-Mult (a shortened version of multifactorial questionnaire for personality research) method to detect certain patterns in clinical and nonclinical samples. 
In patients undergoing treatment, high levels and a tendency towards high levels of hypochondriasis were noted in $33.4 \%$, which is manifested in unsatisfactory health, decreased activity and productivity, need for sympathy, stiffness, exactingness and tension in treatment, neurotic overcontrol.

On the D scale, a significant number of respondents from the clinical sample scored 61-70 T-points (37.5\%) and above 70 T-points (12.5\%), which indicates anxiety and uncertainty, decreased mood, lack of optimism, acute feelings of failure and guilt, sharp disappointment after failure or illness, a tendency to depressive reactions.

On the Hy scale, high scores and a tendency to high scores were recorded in $36.1 \%$ of respondents from the clinical sample. Such people are characterized by high emotional lability, egocentric attitudes and a certain theatricality in behavior. They exaggerate somatic ill-being and deny difficulties in social adaptation. They tend to react to failures and disappointments by developing autonomic and other functional reactions.

A significant number of respondents from the clinical sample noted high $(15.3 \%)$ and with a tendency to high (37.5\%) scores, ranging from 61 to $70 \mathrm{~T}$-points on the psychopathic scale. These prove emotional immaturity of the personality, pronounced impulsivity, a feeling of inability and dissatisfaction with life, injustice and misunderstanding on the part of others.

On the Pa scale, a tendency to high scores was noted in $36.1 \%$ of respondents, high scores were recorded in $12.5 \%$ of respondents, which indicates rigidity, suspicion, resentment, tension of needs, rigidity in the goal implementation. The formation of overvalued ideas (relationships, jealousy) is also possible.

Low frustration tolerance, tension, increased selfcriticism, a tendency to obsessive anxiety, up to the creation of 'ritual behavior' are characteristic of people with high and a tendency to high scores on the psychasthenia scale. Among those subject to treatment, high scores were noted in a significant number of respondents $(43 \%)$.

Emotional coldness, closeness, alienation, specificity of judgments and value systems, difficulties in social adaptation are characteristic of people with high and tendency to high scores on the schizoid scale. In the clinical sample, high scores were recorded in $36.1 \%$ of respondents.

High scores on the hypomanic scale were noted in $20.8 \%$ of respondents, and a tendency to high scores was observed in $27.8 \%$ of respondents, which indicates instability, impulsivity, overactiveness, a tendency to rapid mood changes and to social maladjustment.
The data obtained using the Mini-Mult method for the non-clinical sample differ significantly from the data obtained for healthy individuals.

On the HS scale, a tendency to high and high scores were observed in $18.9 \%$ of respondents.

On the depression scale, $26.4 \%$ of respondents showed high and a tendency to high scores.

High scores and a tendency to high scores on the hysteria scale were noted in a small number of respondents from the non-clinical sample $(5.3 \%$ and $10.5 \%$, respectively).

On the contrary, a significant number of respondents from this sample $(45.3 \%)$ were characterized by low scores and a tendency to low scores on this scale, which indicates emotional stability, confidence, a tendency to focus on oneself in assessing the reality.

A significantly smaller number of respondents from the non-clinical sample $(27.3 \%)$ compared to those from the clinical sample showed high scores and a tendency to high scores on the psychopathic scale.

A similar trend was noted on the paranoia scale $(25.3 \%)$

On the Pt scale, a tendency to high scores was recorded in $12.6 \%$ of respondents from the non-clinical sample, and only $8.4 \%$ of respondents noted high scores, which is significantly less than the indicators in the clinical sample.

On the contrary, a significant number of respondents from the non-clinical sample $(47.4 \%)$ had low scores and exhibited a tendency to low scores on this scale, which shows self-confident and low anxious individuals.

On the Sc scale, a tendency to high scores was noted in $15.8 \%$ of respondents and high scores - in $6.3 \%$ of respondents.

On the Ma scale, the data obtained in the non-clinical sample were not so different from those obtained in the clinical sample. A tendency to high scores was recorded in $27.4 \%$ of respondents and high scores were observed in $15.8 \%$ of respondents.

The nonparametric Mann-Whitney U-test was employed to confirm the statistically significant differences in the MMPI scale indicators of respondents from the clinical and non-clinical samples (Table 1).

As evidenced by the data presented in Table 1, the Mann-Whitney U-test confirmed the statistically significant differences in the MMPI scale indicators of patients undergoing treatment and those who have never sought medical help for mental health problems. The scores for all of these scales are statistically significantly higher in the clinical sample compared to the non-clinical sample. 
Table 1 Comparative analysis of integral indicators in respondents from the clinical and non-clinical samples

\begin{tabular}{|c|c|c|c|c|}
\hline MMPI scales & \multicolumn{2}{|c|}{ Midrank } & $\begin{array}{c}\text { Mann-Whitney U- } \\
\text { test }\end{array}$ & $\begin{array}{c}\text { The level of statistical } \\
\text { significance (p) }\end{array}$ \\
\hline & Clinical sample & Non-clinical sample & 2548.5 & 0.005 \\
\hline $\mathrm{HS}$ & 96.10 & 74.83 & 2444.5 & 0.002 \\
\hline $\mathrm{D}$ & 97.55 & 73.73 & 2449.5 & 0.002 \\
\hline $\mathrm{Hy}$ & 97.48 & 73.78 & 2684.5 & 0.017 \\
\hline $\mathrm{Pd}$ & 94.22 & 76.26 & 2606 & 0.008 \\
\hline $\mathrm{Pa}$ & 95.31 & 75.43 & 2497 & 0.003 \\
\hline $\mathrm{Pt}$ & 96.82 & 74.28 & 2673 & 0.015 \\
\hline
\end{tabular}

sought medical help for mental health problems in terms of their psychoemotional state and personality traits.

\section{CONCLUSION}

Theoretical analysis showed that the emergence of pathological psychoemotional states (neurosis, experience of loneliness, depressive states) is directly related to the social environment and, first of all, to disorders in the system of significant relationships. The dissonance of the personality with significant parts of reality resolved irrationally provokes the emergence of negative experiences.

The development of this process has a specific gradient course: there are no clear topical and temporal boundaries, the qualitative characteristics are plastic and tend to cumulation. The unproductive restoration of the disturbed system of relations causes painful experiences, which lead to psychological and physiological disintegration.

Patients undergoing treatment exhibit a number of features that are less characteristic of those who have never sought medical help for mental health problems.

These features can be conditionally divided into those related to the disharmony of the personality structure and direct psychoemotional manifestations.

The disharmony of the personality structure can manifest itself as a pathological sharpening of personality traits in the form of painful hypochondriasis, emotional lability, combined with demonstrativeness, decreased selfcontrol, impulsivity, emotional coldness, aggressiveness, and impaired social adaptation.

Psychoemotional manifestations include emotional lability and excitability, dissatisfaction with desires, concentration on the experiences of one's own inferiority, decreased activity, the impossibility of emotional relaxation, detachment from reality, as an attempt to adapt to reality that does not correspond to internal ideas.

Thus, the analysis of the data obtained in the empirical study confirmed the hypothesis about differences between patient undergoing treatment and those who have never

\section{REFERENCES}

[1] G. Andersson, J. Bergström, F. Holländare et al., Internet-based self-help for depression: randomised controlled trial, The British J. of Psychiatry 187(5) (2005) 456-461.

[2] P.A. Arean, K.A. Hallgren, J.T. Jordan et al., The use and effectiveness of mobile apps for depression: results from a fully remote clinical trial, J. of Med. Internet Res. 18(12) (2016) 330.

[3] A.A. Bochaver, S.V. Dokuka, E.V. Sivak, I.B. Smirnov, The use of social networks on the Internet and depressive symptoms in adolescents, Clin. Special Psychol. 8(3) (2019) 1-18.

[4] E.A. Gromova, G.V. Kataeva, Yu.G. Khomenko et al., Psychoemotional state and cognitive functions in HIV-infected patients and functional state of brain structures (according to positron emission tomography and magnetic resonance spectroscopy, Clin. Special Psychol. 9(1) (2020) 78-103.

[5] M.M. Danina, N.V. Kiselnikova, E.A. Kuminskaya et al., Depression prevention methods on digital platforms and social media, Clin. Special Psychol. 8(3) (2019) 101-124.

[6] D.Yu. Erokhina, I.V. Zausenko, Experiencing loneliness in people of different ages, Psychol. in a Chang. World: Probl., Hypotheses, Res. (2016) 14-20, South Ural State Univer., Yuzhno-Uralsk.

[7] D.A. Zakharchenko, S.S. Petrikov, Possibilities of using cognitive-behavioral therapy in the correction of depressive and anxiety disorders after a stroke, Consultat. Psychol. and Psychotherapy 26(1) (2018) 95-111.

[8] E.G. Ilchenko, Formation of ideas about neurotic disorders (neuroses), Psychotherapy Bull. 65(70) (2018) 31-48. 
[9] A.I. Knizhnik, Analysis of the problem of the occurrence of depressive states in young people, Intellect. Resources for Reg. Development 1 (2020) 91-95.

[10] S. Køppe, Neurosis: aspects of its conceptual development in the nineteenth century, History of Psychiatry 20( 1) (2009) 27-46.

[11] A. Sheridan, J. Drennan, B. Coughlan et al., Improving social functioning and reducing social isolation and loneliness among people with enduring mental illness: Report of a randomised controlled trial of supported socialization, Int. J. of Social Psychiatry 61(3) (2014) 241-250.

[12] I.M. Slobodchikov, Contemporary research on the experience of loneliness, Psycholog. Sci. and Ed. 12 (3) (2007) 27-35. 